Supplement of Nat. Hazards Earth Syst. Sci., 18, 2093-2109, 2018

https://doi.org/10.5194/nhess-18-2093-2018-supplement

(C) Author(s) 2018. This work is distributed under

the Creative Commons Attribution 4.0 License.

(c) (1)

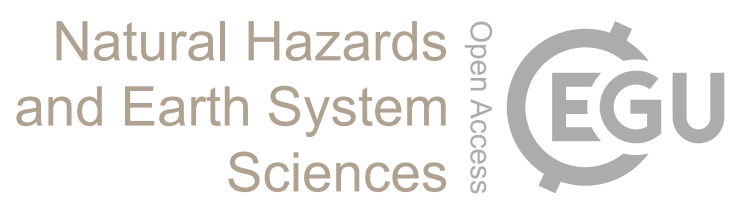

Supplement of

\title{
Natural hazard events affecting transportation networks in Switzerland from 2012 to 2016
}

Jérémie Voumard et al.

Correspondence to: Jérémie Voumard (jeremie.voumard@unil.ch)

The copyright of individual parts of the supplement might differ from the CC BY 4.0 License. 


\section{$1 \quad$ Supplementary Material}

2 Table S1: 51 key words (in red) used in the Google Alerts to create the database. The numbers between 3 brackets in the following tables refer to the number of elements considered according to the line or column 4 attribute.

\begin{tabular}{|c|c|c|c|}
\hline English & French & German & Italian \\
\hline avalanche & avalanche & Lawinne & valanga 6 \\
\hline bad weather & intempéries & Unwetter & \\
\hline flood & & Hochwasser & \\
\hline hail & grêle & Hagel & 7 \\
\hline heavy rainfall & forte pluies & Heftige Regen & \\
\hline ice avalanche & & Eislawine & \\
\hline inundation & & Überflutung & 8 \\
\hline inundation & inondation & Überschwemmung & \\
\hline landslide & glissement de terrain & Erdrutsch & frana \\
\hline landslide & & Hangrutsch & \\
\hline landslide & & Hachrutsche & \\
\hline landslide & & Rüfenniedergang & \\
\hline landslip & glissement & Rutschung & \\
\hline mountain & pan de montagne & & \\
\hline mud & boue & Schlamm & \\
\hline mudflow & coulée de boue & Schlammlawine & \\
\hline mudslide & & Erdlawine & \\
\hline pirock & caillou & Stein & massi \\
\hline rockfall & & Bergsturz & \\
\hline rockfall & & Felsabbruch & \\
\hline rockfall & éboulement & Felsbrock & \\
\hline rockfall & écroulement & Felsbrocken & \\
\hline rockfall & & Felssturz & \\
\hline rockslide & chute de blocs & Steinschlag & cadono sassi \\
\hline scree & & Geröll & \\
\hline scree & éboulis & Schutt & \\
\hline storm & tempête & Sturm & \\
\hline thunderstorm & orage & Gewitter & \\
\hline under water & sous l'eau & & \\
\hline wind & vent & Wind & \\
\hline
\end{tabular}

9

10 Table S2: Cost value estimation by square metre for the cost evaluation according to event importance, 11 damage level and transport mode.

Damage level $\quad$ Cost per $\mathrm{m}^{2}, \quad$ Cost per $\mathrm{m}^{2}, \quad$ Cost per $\mathrm{m}^{2}, \quad$ Cost per $\mathrm{m}^{2}, \quad$ Cost per $\mathrm{m}^{2}, \quad$ Cost per $\mathrm{m}^{2}$, [EUR] small event, middle event, large event, small event, middle event, large event,

\section{2}

\begin{tabular}{lcccccc} 
& road & road & road & train & train & train \\
\hline No closure & 5 & 5 & 5 & 5 & 5 & 5 \\
Closure & 85 & 130 & 170 & 300 & 340 & 385 \\
Partial damage & 255 & 300 & 340 & 470 & 510 & 555 \\
Total destruction & 850 & 890 & 980 & 1065 & 1105 & 1145 \\
Unknown damage & 130 & 170 & 215 & 255 & 300 & 340 \\
\hline
\end{tabular}

13

Table S3: Distribution of event locations by Swiss geomorphologic-climatic region and event process.

\begin{tabular}{lccccccc}
\hline $\begin{array}{l}\text { Geomorphologic- } \\
\text { climatic region }\end{array}$ & $\begin{array}{c}\text { Flood } \\
(420)\end{array}$ & $\begin{array}{c}\text { Debris flow } \\
(69)\end{array}$ & $\begin{array}{c}\text { Landslide } \\
(192)\end{array}$ & $\begin{array}{c}\text { Rockfall } \\
(96)\end{array}$ & $\begin{array}{c}\text { Avalanche } \\
(16)\end{array}$ & $\begin{array}{c}\text { Other } \\
(53)\end{array}$ & Average \\
\hline Jura (98) & $19 \%$ & $0 \%$ & $3 \%$ & $6 \%$ & $0 \%$ & $15 \%$ & $12 \%$ \\
Plateau (371) & $57 \%$ & $4 \%$ & $42 \%$ & $6 \%$ & $0 \%$ & $79 \%$ & $44 \%$ \\
Alps (377) & $24 \%$ & $96 \%$ & $55 \%$ & $88 \%$ & $100 \%$ & $6 \%$ & $44 \%$ \\
Total (846) & $100 \%$ & $100 \%$ & $100 \%$ & $100 \%$ & $100 \%$ & $100 \%$ & $100 \%$ \\
\hline
\end{tabular}

14 
Table S4: Distribution of event locations by event process.

\begin{tabular}{lccccccc}
\hline Event location & $\begin{array}{c}\text { Flood } \\
(420)\end{array}$ & $\begin{array}{c}\text { Debris flow } \\
(69)\end{array}$ & $\begin{array}{c}\text { Landslide } \\
(192)\end{array}$ & $\begin{array}{c}\text { Rockfall } \\
(96)\end{array}$ & $\begin{array}{c}\text { Avalanche } \\
(16)\end{array}$ & $\begin{array}{c}\text { Other } \\
(53)\end{array}$ & Average \\
\hline Town (151) & $15 \%$ & $0 \%$ & $9 \%$ & $1 \%$ & $0 \%$ & $6 \%$ & $18 \%$ \\
Village (261) & $46 \%$ & $14 \%$ & $12 \%$ & $6 \%$ & $13 \%$ & $4 \%$ & $31 \%$ \\
Forest (185) & $4 \%$ & $46 \%$ & $38 \%$ & $58 \%$ & $13 \%$ & $13 \%$ & $22 \%$ \\
Unforested (249) & $0 \%$ & $6 \%$ & $5 \%$ & $12 \%$ & $69 \%$ & $0 \%$ & $29 \%$ \\
Total (846) & $100 \%$ & $100 \%$ & $100 \%$ & $100 \%$ & $100 \%$ & $100 \%$ & $100 \%$ \\
\hline
\end{tabular}

17

Table S5: Distribution of slope angle by event process.

\begin{tabular}{|c|c|c|c|c|c|c|c|}
\hline Slope angle & $\begin{array}{l}\text { Flood } \\
(420)\end{array}$ & $\begin{array}{c}\text { Debris flow } \\
(69)\end{array}$ & $\begin{array}{c}\text { Landslide } \\
\text { (192) }\end{array}$ & $\begin{array}{c}\text { Rockfall } \\
(96)\end{array}$ & $\begin{array}{c}\text { Avalanche } \\
\text { (16) }\end{array}$ & $\begin{array}{l}\text { Other } \\
(53)\end{array}$ & Average \\
\hline $0^{\circ}-10^{\circ}(339)$ & $62 \%$ & $17 \%$ & $12 \%$ & $5 \%$ & $6 \%$ & $68 \%$ & $40 \%$ \\
\hline $10^{\circ}-20^{\circ}(257)$ & $31 \%$ & $43 \%$ & $29 \%$ & $19 \%$ & $38 \%$ & $28 \%$ & $30 \%$ \\
\hline $20^{\circ}-30^{\circ}(131)$ & $4 \%$ & $23 \%$ & $33 \%$ & $31 \%$ & $38 \%$ & $2 \%$ & $15 \%$ \\
\hline $30^{\circ}-40^{\circ}(85)$ & $2 \%$ & $12 \%$ & $21 \%$ & $26 \%$ & $19 \%$ & $0 \%$ & $10 \%$ \\
\hline $40^{\circ}-50^{\circ}(26)$ & $0 \%$ & $4 \%$ & $4 \%$ & $14 \%$ & $0 \%$ & $2 \%$ & $3 \%$ \\
\hline $50^{\circ}-60^{\circ}(6)$ & $0 \%$ & $0 \%$ & $1 \%$ & $4 \%$ & $0 \%$ & $0 \%$ & $1 \%$ \\
\hline 60 and higher (2) & $0 \%$ & $0 \%$ & $1 \%$ & $1 \%$ & $0 \%$ & $0 \%$ & $0 \%$ \\
\hline Total (846) & $100 \%$ & $100 \%$ & $100 \%$ & $100 \%$ & $100 \%$ & $100 \%$ & $100 \%$ \\
\hline
\end{tabular}

Table S6: Distribution of event importance by event process.

\begin{tabular}{|c|c|c|c|c|c|c|c|}
\hline $\begin{array}{l}\text { Location of } \\
\text { process origin }\end{array}$ & $\begin{array}{l}\text { Flood } \\
(420)\end{array}$ & $\begin{array}{l}\text { Debris flow } \\
\text { (69) }\end{array}$ & $\begin{array}{c}\text { Landslide } \\
\text { (192) }\end{array}$ & $\begin{array}{c}\text { Rockfall } \\
\text { (96) }\end{array}$ & $\begin{array}{l}\text { Avalanche } \\
\text { (16) }\end{array}$ & $\begin{array}{l}\text { Other } \\
(53)\end{array}$ & Average \\
\hline Small $^{1}(804)$ & $100 \%$ & $78 \%$ & $96 \%$ & $24 \%$ & $81 \%$ & $100 \%$ & $95 \%$ \\
\hline $\operatorname{Middle}^{2}(33)$ & $0 \%$ & $19 \%$ & $3 \%$ & $43 \%$ & $19 \%$ & $0 \%$ & $4 \%$ \\
\hline $\operatorname{Large}^{3}(9)$ & $0 \%$ & $3 \%$ & $1 \%$ & $33 \%$ & $0 \%$ & $0 \%$ & $1 \%$ \\
\hline Total (846) & $100 \%$ & $100 \%$ & $100 \%$ & $100 \%$ & $100 \%$ & $100 \%$ & $100 \%$ \\
\hline
\end{tabular}
$100 \%$

${ }^{2}$ Middle event: volume of deposit material on the track of $10-2000 \mathrm{~m}^{3}$.

${ }^{3}$ Large event: volume of deposit material on the track $>2000 \mathrm{~m}^{3}$.

Table S7: Distribution of the distance of the process origin by event process.

\begin{tabular}{|c|c|c|c|c|c|c|}
\hline $\begin{array}{l}\text { Distance of the } \\
\text { process origin }\end{array}$ & $\begin{array}{l}\text { Debris flow } \\
\text { (69) }\end{array}$ & $\begin{array}{l}\text { Landslide } \\
\text { (192) }\end{array}$ & $\begin{array}{c}\text { Rockfall } \\
(96)\end{array}$ & $\begin{array}{l}\text { Avalanche } \\
\text { (16) }\end{array}$ & $\begin{array}{l}\text { Other } \\
(53)\end{array}$ & Average \\
\hline Near $^{1}(185)$ & $0 \%$ & $52 \%$ & $33 \%$ & $6 \%$ & $100 \%$ & $35 \%$ \\
\hline $\operatorname{Far}^{2}(146)$ & $100 \%$ & $11 \%$ & $43 \%$ & $94 \%$ & $0 \%$ & $39 \%$ \\
\hline Unknown (95) & $0 \%$ & $37 \%$ & $24 \%$ & $0 \%$ & $0 \%$ & $26 \%$ \\
\hline Total (426) & $100 \%$ & $100 \%$ & $100 \%$ & $100 \%$ & $100 \%$ & $100 \%$ \\
\hline
\end{tabular}

29 Near: $0-50 \mathrm{~m}$ from the track.

$30 \quad{ }^{2}$ Far: $>50 \mathrm{~m}$ from the track.

Table S8: Distribution of the location of the process origin by event process.

\begin{tabular}{lcccccc}
\hline Location of & Debris flow & Landslide & Rockfall & Avalanche & Other \\
process origin & $(69)$ & $(192)$ & $(96)$ & $(16)$ & $(53)$ & Average \\
\hline Above track (339) & $100 \%$ & $60 \%$ & $89 \%$ & $100 \%$ & $100 \%$ & $80 \%$ \\
Below track (29) & $0 \%$ & $14 \%$ & $2 \%$ & $0 \%$ & $0 \%$ & $7 \%$ \\
Unknown (58) & $0 \%$ & $26 \%$ & $9 \%$ & $0 \%$ & $0 \%$ & $14 \%$ \\
Total (426) & $100 \%$ & $100 \%$ & $100 \%$ & $100 \%$ & $100 \%$ & $100 \%$ \\
\hline
\end{tabular}


Table S9: Rainfall [mm] during the natural hazard events.

\begin{tabular}{|c|c|c|c|c|c|c|c|}
\hline Rainfall* [mm] & Flood & Debris flow & Landslide & Rockfall & Avalanche & Other & Average \\
\hline Event day & 22 & 14 & 17 & 5 & 4 & 4 & 17 \\
\hline Cum. last 5 days $^{1}$ & 49 & 32 & 57 & 27 & 32 & 15 & 45 \\
\hline Cum. last 10 days $^{1}$ & 76 & 55 & 88 & 52 & 46 & 36 & 71 \\
\hline Daily rain avg. last 5 days ${ }^{2}$ & 10 & 6 & 11 & 6 & 6 & 3 & 9 \\
\hline Daily rain avg. last 10 days $^{2}$ & 7 & 5 & 9 & 5 & 5 & 4 & 7 \\
\hline Max daily rain last 5 days ${ }^{3}$ & 30 & 21 & 32 & 15 & 18 & 11 & 27 \\
\hline Max daily rain last 10 days $^{3}$ & 33 & 26 & 36 & 20 & 21 & 15 & 30 \\
\hline Abs max daily rain ${ }^{4}$ & 100 & 65 & 154 & 42 & 13 & 39 & - \\
\hline Abs max daily rain last 5 days ${ }^{4}$ & 154 & 75 & 154 & 77 & 140 & 39 & - \\
\hline Abs max daily rain last 10 days $^{4}$ & 154 & 75 & 154 & 109 & 140 & 39 & - \\
\hline
\end{tabular}

A max daily rain last 10 days

154

* Average by event process except for absolute values (last three lines of the table).

$38{ }^{1}$ Cumulative rainfall 5 and 10 days prior to the event day.

392 Daily rainfall average 5 and 10 days prior to the event day.

$40{ }^{3}$ Maximum daily rainfall 5 and 10 days prior to the event day.

$41{ }^{4}$ Absolute maximum rainfall recorded (i.e., for one event) on the event day, 5 and 10 days prior to the event day.

Table S10: Monthly distribution of events by event process.

\begin{tabular}{|c|c|c|c|c|c|c|c|}
\hline Year & $\begin{array}{l}\text { Flood } \\
(420) \\
\end{array}$ & $\begin{array}{c}\text { Debris flow } \\
(69)\end{array}$ & $\begin{array}{c}\text { Landslide } \\
\text { (192) }\end{array}$ & $\begin{array}{c}\text { Rockfall } \\
(96)\end{array}$ & $\begin{array}{c}\text { Avalanche } \\
\text { (16) }\end{array}$ & $\begin{array}{l}\text { Other } \\
(53)\end{array}$ & Average \\
\hline January (27) & $0 \%$ & $4 \%$ & $4 \%$ & $15 \%$ & $6 \%$ & $0 \%$ & $3 \%$ \\
\hline February (65) & $0 \%$ & $1 \%$ & $6 \%$ & $6 \%$ & $19 \%$ & $81 \%$ & $8 \%$ \\
\hline March (26) & $1 \%$ & $0 \%$ & $2 \%$ & $13 \%$ & $50 \%$ & $2 \%$ & $3 \%$ \\
\hline April (28) & $2 \%$ & $0 \%$ & $6 \%$ & $7 \%$ & $0 \%$ & $2 \%$ & $3 \%$ \\
\hline May (107) & $13 \%$ & $10 \%$ & $16 \%$ & $15 \%$ & $0 \%$ & $2 \%$ & $13 \%$ \\
\hline June (253) & $41 \%$ & $16 \%$ & $29 \%$ & $7 \%$ & $0 \%$ & $8 \%$ & $30 \%$ \\
\hline July (210) & $31 \%$ & $51 \%$ & $19 \%$ & $8 \%$ & $0 \%$ & $2 \%$ & $25 \%$ \\
\hline August (35) & $4 \%$ & $12 \%$ & $4 \%$ & $1 \%$ & $0 \%$ & $2 \%$ & $4 \%$ \\
\hline September (14) & $1 \%$ & $6 \%$ & $2 \%$ & $2 \%$ & $0 \%$ & $0 \%$ & $2 \%$ \\
\hline October (14) & $1 \%$ & $0 \%$ & $1 \%$ & $10 \%$ & $0 \%$ & $0 \%$ & $2 \%$ \\
\hline November (58) & $6 \%$ & $0 \%$ & $9 \%$ & $11 \%$ & $6 \%$ & $2 \%$ & $7 \%$ \\
\hline December (9) & $0 \%$ & $0 \%$ & $1 \%$ & $4 \%$ & $19 \%$ & $0 \%$ & $1 \%$ \\
\hline Total (846) & $100 \%$ & $100 \%$ & $100 \%$ & $100 \%$ & $100 \%$ & $100 \%$ & $100 \%$ \\
\hline
\end{tabular}

44

45

$46 \quad$ Table S11: Transport mode distribution by event process.

\begin{tabular}{lccccccc} 
Transport mode & $\begin{array}{c}\text { Flood } \\
(420)\end{array}$ & $\begin{array}{c}\text { Debris flow } \\
(69)\end{array}$ & $\begin{array}{c}\text { Landslide } \\
(192)\end{array}$ & $\begin{array}{c}\text { Rockfall } \\
(96)\end{array}$ & $\begin{array}{c}\text { Avalanche } \\
(16)\end{array}$ & $\begin{array}{c}\text { Other } \\
(53)\end{array}$ & Total \\
\hline Road (747) & $53 \%$ & $9 \%$ & $20 \%$ & $10 \%$ & $1 \%$ & $7 \%$ & $100 \%$ \\
Railway (99) & $27 \%$ & $2 \%$ & $42 \%$ & $20 \%$ & $4 \%$ & $5 \%$ & $100 \%$ \\
\hline
\end{tabular}

47

Table S12: Road class distribution by event process.

\begin{tabular}{lccccccc}
\hline Road class & $\begin{array}{c}\text { Flood } \\
(393)\end{array}$ & $\begin{array}{c}\text { Debris flow } \\
(67)\end{array}$ & $\begin{array}{c}\text { Landslide } \\
(151)\end{array}$ & $\begin{array}{c}\text { Rockfall } \\
(76)\end{array}$ & $\begin{array}{c}\text { Avalanche } \\
(12)\end{array}$ & $\begin{array}{c}\text { Other } \\
(48)\end{array}$ & $\begin{array}{c}\text { Average } \\
\text { Highway (34) }\end{array}$ \\
\hline Motorway (2) & $0 \%$ & $0 \%$ & $2 \%$ & $1 \%$ & $10 \%$ & $2 \%$ & $5 \%$ \\
Major transit road (99) & $11 \%$ & $8 \%$ & $1 \%$ & $0 \%$ & $0 \%$ & $0 \%$ & $0 \%$ \\
Regional road (94) & $11 \%$ & $7 \%$ & $11 \%$ & $36 \%$ & $36 \%$ & $6 \%$ & $13 \%$ \\
Urban road (426) & $65 \%$ & $37 \%$ & $48 \%$ & $38 \%$ & $36 \%$ & $82 \%$ & $57 \%$ \\
Minor road (72) & $4 \%$ & $42 \%$ & $15 \%$ & $4 \%$ & $9 \%$ & $2 \%$ & $10 \%$ \\
Forest or land trail (20) & $2 \%$ & $6 \%$ & $5 \%$ & $5 \%$ & $0 \%$ & $0 \%$ & $3 \%$ \\
Total (747) & $100 \%$ & $100 \%$ & $100 \%$ & $100 \%$ & $100 \%$ & $100 \%$ & $100 \%$ \\
\hline
\end{tabular}


Table S13: Railway class distribution by event process.

\begin{tabular}{lccccccc}
\hline Track class & $\begin{array}{c}\text { Flood } \\
(27)\end{array}$ & $\begin{array}{c}\text { Debris flow } \\
(2)\end{array}$ & $\begin{array}{c}\text { Landslide } \\
(41)\end{array}$ & $\begin{array}{c}\text { Rockfall } \\
(20)\end{array}$ & $\begin{array}{c}\text { Avalanche } \\
(4)\end{array}$ & $\begin{array}{c}\text { Other } \\
(5)\end{array}$ & Average \\
\hline National (29) & $37 \%$ & $0 \%$ & $32 \%$ & $30 \%$ & $0 \%$ & $0 \%$ & $29 \%$ \\
Regional (66) & $56 \%$ & $100 \%$ & $68 \%$ & $70 \%$ & $100 \%$ & $60 \%$ & $67 \%$ \\
Tram (4) & $7 \%$ & $0 \%$ & $0 \%$ & $0 \%$ & $0 \%$ & $40 \%$ & $4 \%$ \\
Total (99) & $100 \%$ & $100 \%$ & $100 \%$ & $100 \%$ & $100 \%$ & $100 \%$ & $100 \%$ \\
\hline
\end{tabular}

Table S14: Distribution of possibility of deviations by event process.

\begin{tabular}{lccccccc}
\hline Possibility of deviation & $\begin{array}{c}\text { Flood } \\
(420)\end{array}$ & $\begin{array}{c}\text { Debris flow } \\
(69)\end{array}$ & $\begin{array}{c}\text { Landslide } \\
(192)\end{array}$ & $\begin{array}{c}\text { Rockfall } \\
(96)\end{array}$ & $\begin{array}{c}\text { Avalanche } \\
(16)\end{array}$ & $\begin{array}{c}\text { Other } \\
(53)\end{array}$ & Total \\
\hline Large (342) & $63 \%$ & $17 \%$ & $15 \%$ & $8 \%$ & $0 \%$ & $52 \%$ & $40 \%$ \\
Middle (190) & $21 \%$ & $7 \%$ & $32 \%$ & $17 \%$ & $7 \%$ & $33 \%$ & $23 \%$ \\
Small (102) & $7 \%$ & $6 \%$ & $13 \%$ & $32 \%$ & $66 \%$ & $4 \%$ & $12 \%$ \\
No (212) & $9 \%$ & $70 \%$ & $40 \%$ & $43 \%$ & $27 \%$ & $11 \%$ & $25 \%$ \\
Total (846) & $100 \%$ & $100 \%$ & $100 \%$ & $100 \%$ & $100 \%$ & $100 \%$ & $100 \%$ \\
\hline
\end{tabular}

\section{5}

Total (846)

$56 \quad$ Table S15: Distribution of track damage by event process.

\begin{tabular}{lccccccc}
\hline Damage level & $\begin{array}{c}\text { Flood } \\
(420)\end{array}$ & $\begin{array}{c}\text { Debris flow } \\
(69)\end{array}$ & $\begin{array}{c}\text { Landslide } \\
(192)\end{array}$ & $\begin{array}{c}\text { Rockfall } \\
(96)\end{array}$ & $\begin{array}{c}\text { Avalanche } \\
(16)\end{array}$ & $\begin{array}{c}\text { Other } \\
(53)\end{array}$ & Total \\
\hline No closure (149) & $34 \%$ & $0 \%$ & $1 \%$ & $3 \%$ & $6 \%$ & $4 \%$ & $18 \%$ \\
Closure (483) & $60 \%$ & $35 \%$ & $50 \%$ & $50 \%$ & $81 \%$ & $96 \%$ & $57 \%$ \\
Partial damage (143) & $1 \%$ & $39 \%$ & $37 \%$ & $39 \%$ & $13 \%$ & $0 \%$ & $17 \%$ \\
Total destruction (53) & $1 \%$ & $26 \%$ & $12 \%$ & $8 \%$ & $0 \%$ & $0 \%$ & $6 \%$ \\
Unknown damage (18) & $4 \%$ & $0 \%$ & $0 \%$ & $0 \%$ & $0 \%$ & $0 \%$ & $2 \%$ \\
Total (846) & $100 \%$ & $100 \%$ & $100 \%$ & $100 \%$ & $100 \%$ & $100 \%$ & $100 \%$ \\
\hline
\end{tabular}

Table S16: Distribution of damage and impact on vehicles by event process.

Damage and impact type on vehicles Flood Debris flow Landslide Rockfall

\begin{tabular}{|c|c|c|c|c|c|c|c|}
\hline & $(420)$ & $(69)$ & $(192)$ & $(96)$ & $(16)$ & $(53)$ & \\
\hline No damage (803) & $98 \%$ & $93 \%$ & $96 \%$ & $89 \%$ & $80 \%$ & $89 \%$ & $95 \%$ \\
\hline Vehicle damage: direct impact ${ }^{1}(25)$ & $1 \%$ & $7 \%$ & $1 \%$ & $7 \%$ & $7 \%$ & $7 \%$ & $3 \%$ \\
\hline Vehicle damage: indirect impact ${ }^{2}(18)$ & $1 \%$ & $0 \%$ & $3 \%$ & $4 \%$ & $13 \%$ & $4 \%$ & $2 \%$ \\
\hline Total $(846)$ & $100 \%$ & $100 \%$ & $100 \%$ & $100 \%$ & $100 \%$ & $100 \%$ & $100 \%$ \\
\hline
\end{tabular}

${ }^{1}$ Direct impact: a vehicle is directly affected by a hazard.

602 Indirect impact: a vehicle collides with an event mass already fallen on the track.

62 Table S17: Distribution of injury and death by event process.

\begin{tabular}{lccccccc}
\hline Injury and death & Flood & Debris flow & Landslide & Rockfall & Avalanche & Other & Total \\
& $(420)$ & $(69)$ & $(192)$ & $(96)$ & $(16)$ & $(53)$ \\
\hline No damage on people (828) & $99 \%$ & $96 \%$ & $98 \%$ & $93 \%$ & $100 \%$ & $98 \%$ & $98 \%$ \\
Injury (15) & $1 \%$ & $4 \%$ & $1 \%$ & $5 \%$ & $0 \%$ & $2 \%$ & $2 \%$ \\
Death (3) & $0 \%$ & $0 \%$ & $1 \%$ & $2 \%$ & $0 \%$ & $0 \%$ & $0 \%$ \\
Total (846) & $100 \%$ & $100 \%$ & $100 \%$ & $100 \%$ & $100 \%$ & $100 \%$ & $100 \%$ \\
\hline
\end{tabular}

63

64 Table S18: Distribution of deviation length on roads by event process.

\begin{tabular}{lccccccc}
\hline Deviation length & $\begin{array}{c}\text { Flood } \\
(383)\end{array}$ & $\begin{array}{c}\text { Debris flow } \\
(21)\end{array}$ & $\begin{array}{c}\text { Landslide } \\
(116)\end{array}$ & $\begin{array}{c}\text { Rockfall } \\
(58)\end{array}$ & $\begin{array}{c}\text { Avalanche } \\
(11)\end{array}$ & $\begin{array}{c}\text { Other } \\
(49)\end{array}$ & Mean \\
\hline $0-1 \mathrm{~km}(255)$ & $58 \%$ & $29 \%$ & $12 \%$ & $9 \%$ & $0 \%$ & $12 \%$ & $40 \%$ \\
$2-5 \mathrm{~km}(102)$ & $14 \%$ & $38 \%$ & $16 \%$ & $3 \%$ & $0 \%$ & $39 \%$ & $16 \%$ \\
$6-9 \mathrm{~km}(57)$ & $9 \%$ & $10 \%$ & $9 \%$ & $7 \%$ & $0 \%$ & $14 \%$ & $9 \%$ \\
$10-19 \mathrm{~km}(100)$ & $9 \%$ & $5 \%$ & $34 \%$ & $21 \%$ & $0 \%$ & $22 \%$ & $16 \%$ \\
$20-49 \mathrm{~km}(63)$ & $5 \%$ & $0 \%$ & $17 \%$ & $26 \%$ & $45 \%$ & $8 \%$ & $10 \%$ \\
$50-99 \mathrm{~km}(24)$ & $3 \%$ & $5 \%$ & $5 \%$ & $12 \%$ & $0 \%$ & $0 \%$ & $4 \%$ \\
$100-249 \mathrm{~km}(30)$ & $2 \%$ & $14 \%$ & $6 \%$ & $17 \%$ & $18 \%$ & $4 \%$ & $5 \%$ \\
$250-350 \mathrm{~km}(7)$ & $0 \%$ & $0 \%$ & $0 \%$ & $5 \%$ & $36 \%$ & $0 \%$ & $1 \%$ \\
Total (638) & $100 \%$ & $100 \%$ & $100 \%$ & $100 \%$ & $100 \%$ & $100 \%$ & $100 \%$ \\
\hline
\end{tabular}


Table S19: Direct damage cost distribution by events type.

\begin{tabular}{lccccccc}
\hline Damage level [EUR] & $\begin{array}{c}\text { Flood } \\
(420)\end{array}$ & $\begin{array}{c}\text { Debris } \\
\text { flow (69) }\end{array}$ & $\begin{array}{c}\text { Landslide } \\
(192)\end{array}$ & $\begin{array}{c}\text { Rockfall } \\
(96)\end{array}$ & $\begin{array}{c}\text { Avalanche } \\
(16)\end{array}$ & $\begin{array}{c}\text { Other } \\
(53)\end{array}$ & Total \\
\cline { 2 - 8 } & \multicolumn{7}{c}{ Annual cost [EUR] } \\
\hline No closure (149) & 12665 & 340 & 85 & 765 & 255 & 170 & 14280 \\
Closure (483) & 514250 & 71400 & 262650 & 160650 & 28900 & 107950 & 1145800 \\
Partial damage (143) & 25500 & 127500 & 425000 & 227800 & 40800 & 0 & 846600 \\
Total destruction (53) & 72250 & 459850 & 528700 & 246500 & 0 & 0 & 1307300 \\
Unknown damage (18) & 45900 & 0 & 0 & 0 & 0 & 0 & 45900 \\
Annual cost [million $€$ ] & 0.67 & 0.66 & 1.22 & 0.64 & 0.07 & 0.11 & 3.36 \\
Avg. cost by event & 8000 & 47800 & 31700 & 33100 & 21900 & 10200 & 19900 \\
\hline
\end{tabular}

68

$\begin{array}{lllll}\text { Avg. cost by event } & 8000 & 47800 & 31700 & 33100\end{array}$

69 Table S20: Annual distribution of events by event process.

\begin{tabular}{lccccccc}
\hline Year & $\begin{array}{c}\text { Flood } \\
(420)\end{array}$ & $\begin{array}{c}\text { Debris flow } \\
(69)\end{array}$ & $\begin{array}{c}\text { Landslide } \\
(192)\end{array}$ & $\begin{array}{c}\text { Rockfall } \\
(96)\end{array}$ & $\begin{array}{c}\text { Avalanche } \\
(16)\end{array}$ & $\begin{array}{c}\text { Other } \\
(53)\end{array}$ & Average \\
\hline $2012(60)$ & $5 \%$ & $3 \%$ & $7 \%$ & $17 \%$ & $25 \%$ & $2 \%$ & $7 \%$ \\
$2013(99)$ & $11 \%$ & $10 \%$ & $16 \%$ & $14 \%$ & $6 \%$ & $2 \%$ & $12 \%$ \\
$2014(173)$ & $20 \%$ & $10 \%$ & $30 \%$ & $20 \%$ & $25 \%$ & $0 \%$ & $20 \%$ \\
$2015(245)$ & $25 \%$ & $49 \%$ & $22 \%$ & $17 \%$ & $25 \%$ & $77 \%$ & $29 \%$ \\
$2016(269)$ & $38 \%$ & $28 \%$ & $24 \%$ & $33 \%$ & $19 \%$ & $19 \%$ & $32 \%$ \\
Total $(846)$ & $100 \%$ & $100 \%$ & $100 \%$ & $100 \%$ & $100 \%$ & $100 \%$ & $100 \%$ \\
\hline
\end{tabular}

70

71 
Table S21: Summary of event process key features.

\begin{tabular}{|c|c|c|c|c|c|c|c|}
\hline $\begin{array}{l}\text { Attribute (with } \\
\text { values of the } \\
\text { greatest } \\
\text { occurrence) }\end{array}$ & Flood & Debris flow & Landslide & Rockfall & Avalanche & Other & Mean \\
\hline Event importance & Small & Small & Small & Small & Small & Small & Small \\
\hline $\begin{array}{l}\text { Yearly number of } \\
\text { events }\end{array}$ & 84 & 14 & 38 & 19 & 3 & 11 & 169 \\
\hline Months & 6,7 & 7,6 & $6,7,5$ & $\begin{array}{l}1,5,3,11 \\
10\end{array}$ & 3 & 2 & 6,7 \\
\hline Season & Spring & Summer & Spring & $\begin{array}{l}\text { Spring, } \\
\text { Winter }\end{array}$ & Winter & Winter & Spring \\
\hline Time of day & Afternoon & Afternoon & All day & All day & Morning & All day & Afternoon \\
\hline Hour & $12-19$ & $15-19$ & $0-24$ & $0-24$ & $8-13$ & $0-24$ & $14-19$ \\
\hline Region & Plateau & Alps & Alps & Alps & Alps & Plateau & $\begin{array}{l}\text { Alps, } \\
\text { Plateau }\end{array}$ \\
\hline Canton & Bern & Graubünden & Valais & Valais & Valais & Vaud & Bern \\
\hline Slope angle & $0-10$ & $10-20$ & $20-30$ & $20-30$ & $10-20$ & $0-10$ & $0-10$ \\
\hline Slope orientation & $\mathrm{S}$ & $\mathrm{W}$ & S & $\mathrm{W}$ & $\mathrm{N}-\mathrm{W}$ & S-E & $\begin{array}{l}\text { S, S-W and } \\
\text { W }\end{array}$ \\
\hline Location & Village & Forest & Forest & Forest & Mountain & Country & Village \\
\hline Damage on track & Closure & Partial dam. & Closure & Closure & Closure & Closure & Closure \\
\hline $\begin{array}{l}\text { Direct costs per } \\
\text { event (Euro) }\end{array}$ & 6900 & 39000 & 25700 & 261000 & 155000 & 8600 & 16000 \\
\hline Track geometry & Str. line & Wide curve & Wide curve & Wide curve & Wide curve & $\begin{array}{l}\text { S. line \& w. } \\
\text { curve }\end{array}$ & Wide curve \\
\hline Crossing & Near & No & No & No & No & No & No \\
\hline Closure duration & 3 hours & 1 week & 1 day & 3 hours & 1-2 days & 3 hours & 3 hours \\
\hline $\begin{array}{l}\text { Possibility of } \\
\text { deviation }\end{array}$ & Large & No & No & No & Small & Middle & Large \\
\hline Deviation length & $0-1 \mathrm{~km}$ & No deviation & No deviation & No deviation & $250-350 \mathrm{~km}$ & $2-5 \mathrm{~km}$ & $0-1 \mathrm{~km}$ \\
\hline $\begin{array}{l}\text { Event origin } \\
\text { distance }\end{array}$ & - & Far & Near & Far & Far & Near & Near \\
\hline $\begin{array}{l}\text { Event above } \\
\text { below }\end{array}$ & - & Up & $\mathrm{Up}$ & Up & Up & Up & Up \\
\hline Altitude [m a.s.1.] & 525 & 1139 & 809 & 897 & 1274 & 614 & 701 \\
\hline Track type & Road & Road & Road & Road & Road & Road & Road \\
\hline Track importance & Minor & Minor & Minor & Minor & Minor & Minor & Minor \\
\hline $\begin{array}{l}\text { Rainfall event } \\
\text { day [mm] }\end{array}$ & 22 & 14 & 171 & 5 & 4 & 4 & 17 \\
\hline
\end{tabular}

73 
Figure S1: Attributes of the database.

\begin{tabular}{|c|c|c|c|c|c|c|c|c|c|c|c|c|c|c|c|c|}
\hline \multirow{2}{*}{$\begin{array}{l}\text { Category } \\
\text { Attribute }\end{array}$} & \multirow[b]{2}{*}{ Eventid } & \multicolumn{15}{|c|}{ DATE } \\
\hline & & D_IDdate & D_year & D_Month & D_Day & $\begin{array}{c}\text { D_MonthWe } \\
\text { ek }\end{array}$ & D_DayName & D_Season & D_Hour & $\begin{array}{c}\text { D_HourPreci } \\
\text { se }\end{array}$ & D_DayPart & D_IDDay & $\begin{array}{c}\text { D_IDEventsa } \\
\text { meDay }\end{array}$ & $\begin{array}{l}\text { D_SameClim } \\
\text { LongPeriod }\end{array}$ & $\begin{array}{l}\text { D_SameClim } \\
\text { ShortPeriod }\end{array}$ & MuenichRe \\
\hline Description & $\begin{array}{l}\text { Unique ID } \\
\text { for each } \\
\text { event }\end{array}$ & $\begin{array}{l}\text { Unique ID } \\
\text { for each } \\
\text { event } \\
\text { containing } \\
\text { the date }\end{array}$ & $\begin{array}{c}\text { Year of the } \\
\text { event }\end{array}$ & $\begin{array}{c}\text { Month of the } \\
\text { event }\end{array}$ & $\begin{array}{l}\text { Day of the } \\
\text { event }\end{array}$ & $\begin{array}{c}\text { Month } \\
\text { divided into } \\
4 \text { quarters }\end{array}$ & $\begin{array}{c}\begin{array}{c}\text { Name of the } \\
\text { day of the } \\
\text { event }\end{array}\end{array}$ & $\begin{array}{l}\text { Season of } \\
\text { the event }\end{array}$ & $\begin{array}{c}\begin{array}{c}\text { Hour of the } \\
\text { event hourly } \\
\text { rounded }\end{array} \\
\end{array}$ & $\begin{array}{c}\text { Hour of the } \\
\text { event }\end{array}$ & $\begin{array}{l}\text { Day part of } \\
\text { the event }\end{array}$ & $\begin{array}{c}\text { Unique ID } \\
\text { for each } \\
\text { event day } \\
\text { (same ID } \\
\text { when }>1 \\
\text { event per } \\
\text { day) } \\
\end{array}$ & $\begin{array}{c}\text { Unique ID } \\
\text { for event } \\
\text { occured the } \\
\text { same day }\end{array}$ & $\begin{array}{c}\text { Long time } \\
\text { period in } \\
\text { which he } \\
\text { event is } \\
\text { included }\end{array}$ & $\begin{array}{c}\text { Short time } \\
\text { period in } \\
\text { which the } \\
\text { event is } \\
\text { included }\end{array}$ & $\begin{array}{c}\text { Period given } \\
\text { by MunnichRe } \\
\text { in which the } \\
\text { event is } \\
\text { included }\end{array}$ \\
\hline Unit & - & $y m d x x$ & year & month & day & - & - & - & $h: m: s$ & h:m:s & - & ymd & - & y.m.d-y.m.d & y.m.d-y.m.d & y.m.d-y.m.d.d \\
\hline Exemple & 431 & 2015050400 & 2015 & 5 & 4 & 5-1 & Monday & Spring & 10:00:00 & 10:15:00 & Morning & 20150504 & 2 & $\begin{array}{c}2015.04 .27 . \\
2015.07 .25\end{array}$ & $\begin{array}{l}2015.04 .27 \\
2015.05 .07\end{array}$ & $\begin{array}{l}2014.06 .03 . \\
2014.06 .12\end{array}$ \\
\hline Comment & - & - & $\begin{array}{c}\text { From } 2011 \text { to } \\
2015\end{array}$ & - & - & $\begin{array}{l}\text { First quarter } \\
\text { (1) of the 5th } \\
\text { month (5) }\end{array}$ & $\begin{array}{c}\text { Useful to } \\
\text { categorise } \\
\text { business day } \\
\text { and } \\
\text { weekend }\end{array}$ & - & - & & $\begin{array}{l}5 \text { parts: } \\
\text { morning, } \\
\text { afternoon, } \\
\text { evening, } \\
\text { night and } \\
\text { unknown }\end{array}$ & $\begin{array}{l}\text { Allow to } \\
\text { recognise } \\
\text { the day } \\
\text { when with } \\
\text { several } \\
\text { events }\end{array}$ & $\begin{array}{l}\text { The maximal } \\
\text { ID by event } \\
\text { day gives the } \\
\text { nb of events } \\
\text { during this } \\
\text { day }\end{array}$ & & & \begin{tabular}{|c|} 
From \\
MuenichRe \\
yearly \\
natural \\
catastrophes \\
analysis \\
\end{tabular} \\
\hline Source & 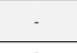 & - & $\begin{array}{l}\text { Online } \\
\text { article }\end{array}$ & $\begin{array}{l}\text { Online } \\
\text { article }\end{array}$ & $\begin{array}{l}\text { Online } \\
\text { article }\end{array}$ & $\begin{array}{l}\text { Online } \\
\text { article }\end{array}$ & $\begin{array}{l}\text { Online } \\
\text { article }\end{array}$ & $\begin{array}{l}\text { Online } \\
\text { article }\end{array}$ & $\begin{array}{l}\text { Online } \\
\text { article }\end{array}$ & $\begin{array}{l}\text { Online } \\
\text { article }\end{array}$ & $\begin{array}{l}\text { Online } \\
\text { article }\end{array}$ & - & - & - & 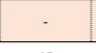 & Münichre \\
\hline
\end{tabular}

\begin{tabular}{|c|c|c|c|c|c|c|c|c|c|c|}
\hline \multirow{3}{*}{$\begin{array}{l}\text { Category } \\
\text { Attribute }\end{array}$} & \\
\hline & & & & & & & & & & \\
\hline & L_Canton & L_commune & L_Detail & L_Precision & L__sitGeo & L_orislope & L_Urbanity & L_Slope & $\begin{array}{c}\text { L_Sloperoun } \\
d\end{array}$ & L_Lanscape \\
\hline Description & $\begin{array}{c}\text { Canton } \\
\text { where } \\
\text { occurs the } \\
\text { event }\end{array}$ & $\begin{array}{c}\text { Commune } \\
\text { where } \\
\text { occurs the } \\
\text { event }\end{array}$ & $\begin{array}{l}\text { Detail to } \\
\text { help the } \\
\text { location }\end{array}$ & $\begin{array}{l}\text { Precision of } \\
\text { the location }\end{array}$ & $\begin{array}{c}\text { Geographical } \\
\text { situation of } \\
\text { the event }\end{array}$ & $\begin{array}{l}\text { If slope: } \\
\text { orientation } \\
\text { of the slope }\end{array}$ & $\begin{array}{l}\text { Urbanity of } \\
\text { the event }\end{array}$ & $\begin{array}{c}\begin{array}{c}\text { Slope angle } \\
\text { average in } \\
\text { an } 25 \text { meter } \\
\text { radius } \\
\text { around the } \\
\text { event }\end{array} \\
\end{array}$ & $\begin{array}{c}\text { Slope angle } \\
\text { rounded to } \\
\text { the nearest } \\
\text { ten }\end{array}$ & $\begin{array}{l}\text { Lanscape of } \\
\text { the event } \\
\text { locaiotn }\end{array}$ \\
\hline Unit & . & $\cdot$ & . & $\cdot$ & . & . & $\cdot$ & {$\left[l^{\circ}\right]$} & {$[1]$} & \\
\hline Exemple & Valais & Bagnes & $\cdot$ & Accurate & Slope & North-East & Forest & 13 & 13 & $\begin{array}{l}\text { Dry } \\
\text { mountainou } \\
\text { s landscape } \\
\text { of western } \\
\text { central Alps }\end{array}$ \\
\hline Comment & - & - & - & $\begin{array}{l}\text { Three levels } \\
\text { of accuracy: } \\
\text { accurate, } \\
\text { middle and } \\
\text { communal } \\
\text { accuracy }\end{array}$ & $\begin{array}{c}\text { Four classes: } \\
\text { plain, ridge, } \\
\text { slope and } \\
\text { valley } \\
\text { bottom }\end{array}$ & $\begin{array}{l}\text { Nine classes: } \\
\text { north, north- } \\
\text { east, south- } \\
\text { east, south, } \\
\text { south-west, } \\
\text { west, noth- } \\
\text { west and any } \\
\text { slope }\end{array}$ & $\begin{array}{c}\text { Seven } \\
\text { classes: } \\
\text { mountain, } \\
\text { forest, } \\
\text { country, } \\
\text { hamlet, } \\
\text { village, } \\
\text { agglomerati } \\
\text { on and town }\end{array}$ & $\begin{array}{c}\text { From } 0^{\circ} \text { to } \\
56^{\circ}\end{array}$ & $\begin{array}{c}\text { From } 0^{\circ} \text { to } \\
60^{\circ}\end{array}$ & 36 types \\
\hline Source & $\begin{array}{l}\text { Online } \\
\text { article }\end{array}$ & $\begin{array}{l}\text { Online } \\
\text { article }\end{array}$ & $\begin{array}{l}\text { Online } \\
\text { article }\end{array}$ & $\begin{array}{l}\text { Online } \\
\text { article and } \\
\text { map }\end{array}$ & Map & Map & Map & GIS & GIS & GIS \\
\hline & 17 & 18 & 19 & 20 & 21 & 22 & 23 & 24 & 25 & 26 \\
\hline
\end{tabular}




\begin{tabular}{|c|c|c|c|c|c|c|c|c|c|c|}
\hline \multicolumn{11}{|l|}{ LOCATION } \\
\hline L_Areas & L_Area_reg & L_MNO3_X & L_MNO3_Y & L_MNO3_Z & L_MN95_X & L_MN95_Y & L_MN95_Z & L_WGS84_LO & L_WGS84_La & L_WGS84_Z \\
\hline $\begin{array}{c}\text { Areas of the } \\
\text { event } \\
\text { location }\end{array}$ & $\begin{array}{l}\text { Regional } \\
\text { area of the } \\
\text { location }\end{array}$ & $\begin{array}{c}\mathrm{X} \\
\text { coordinates } \\
\text { in CH1903 } \\
\text { coordinate } \\
\text { system }\end{array}$ & $\begin{array}{c}Y \\
\text { coordinates } \\
\text { in CH1903 } \\
\text { coordinate } \\
\text { system }\end{array}$ & $\begin{array}{c}\mathrm{Z} \\
\text { coordinates } \\
\text { in CH1903 } \\
\text { coordinate } \\
\text { system }\end{array}$ & $\begin{array}{c}\mathrm{X} \\
\text { coordinates } \\
\text { in CH1903+ } \\
\text { coordinate } \\
\text { system }\end{array}$ & $\begin{array}{c}Y \\
\text { coordinates } \\
\text { in CH1903+ } \\
\text { coordinate } \\
\text { system }\end{array}$ & $\begin{array}{c}\mathrm{Z} \\
\text { coordinates } \\
\text { in CH1903+ } \\
\text { coordinate } \\
\text { system }\end{array}$ & $\begin{array}{l}\text { Longitude in } \\
\text { WGS } 84 \\
\text { coordinate } \\
\text { system }\end{array}$ & $\begin{array}{l}\text { Latitude in } \\
\text { WGS } 84 \\
\text { coordinate } \\
\text { system }\end{array}$ & $\begin{array}{l}\text { ALtitude in } \\
\text { WGS84 } \\
\text { coordinate } \\
\text { system }\end{array}$ \\
\hline & & [m] & {$[\mathrm{m}]$} & {$[\mathrm{m}]$} & {$[\mathrm{m}]$} & {$[\mathrm{m}]$} & {$[\mathrm{m}]$} & {$\left[{ }^{\circ}\right]$} & {$\left[{ }^{\circ}\right]$} & {$[\mathrm{m}]$} \\
\hline $\begin{array}{l}\text { Alpine } \\
\text { region }\end{array}$ & Alps & 588456 & 98247 & 1377 & 2588455 & 1098247 & 1377 & 7.289538659 & 46.03566307 & 1431 \\
\hline $\begin{array}{l}5 \text { types: } \\
\text { Alpine } \\
\text { region, } \\
\text { Swiss } \\
\text { Plateau, } \\
\text { Tabular Jura, } \\
\text { Folded Jura } \\
\text { and } \\
\text { Independent }\end{array}$ & $\begin{array}{c}3 \text { types: Jura, } \\
\text { Plateau and } \\
\text { Alps }\end{array}$ & . & - & - & - & - & - & - & - & - \\
\hline GIS & Map & GIS & GIS & GIS & GIS & GIS & GIS & GIS & GIS & GIS \\
\hline 27 & 28 & 29 & 30 & 31 & 32 & 33 & 34 & 35 & 36 & 37 \\
\hline
\end{tabular}

\begin{tabular}{|c|c|c|c|c|c|c|c|c|c|c|c|c|}
\hline \multirow{3}{*}{$\begin{array}{l}\text { Category } \\
\text { Attribute }\end{array}$} & \multicolumn{12}{|c|}{ nt characterizati } \\
\hline & \multicolumn{12}{|c|}{ Event characterization } \\
\hline & E_Type & E_TypePrec & E_UpDownst & $\begin{array}{c}\text { E_UpDownst } \\
\text { Risk }\end{array}$ & E_Provenan & E_Volume & E_Masse & E_Width & E_Importan & E_other & $\begin{array}{c}\text { E_Picture Na } \\
\text { me }\end{array}$ & E_picture \\
\hline Description & $\begin{array}{c}\text { Type of } \\
\text { natural } \\
\text { hazard event }\end{array}$ & $\begin{array}{c}\text { Precise type } \\
\text { of natural } \\
\text { hazard event }\end{array}$ & $\begin{array}{c}\text { Origin up or } \\
\text { downstream } \\
\text { of the } \\
\text { natural } \\
\text { hazard event }\end{array}$ & $\begin{array}{c}\text { Origin up, } \\
\text { downstream } \\
\text { or only risk } \\
\text { of the event }\end{array}$ & $\begin{array}{c}\text { Estimation } \\
\text { of the } \\
\text { distance of } \\
\text { the event } \\
\text { origin }\end{array}$ & $\begin{array}{l}\text { Volume of } \\
\text { the event }\end{array}$ & $\begin{array}{c}\text { Masse of the } \\
\text { event }\end{array}$ & $\begin{array}{l}\text { Width of the } \\
\text { event mass } \\
\text { on the track }\end{array}$ & $\begin{array}{l}\text { Importance } \\
\text { of the event }\end{array}$ & $\begin{array}{c}\text { Other } \\
\text { information }\end{array}$ & $\begin{array}{c}\text { Picture } \\
\text { name of the } \\
\text { event }\end{array}$ & Picture \\
\hline Unit & - & - & - & - & {$[\mathrm{m}]$ or - } & {$\left[\mathrm{m}^{3}\right]$} & [kg] & {$[\mathrm{m}]$} & - & - & $\cdot$ & - \\
\hline Exemple & Landslide & Landslide & - & - & - & - & - & - & Small & - & $\begin{array}{c}2015050400 . j \\
p g\end{array}$ & - \\
\hline Comment & $\begin{array}{c}6 \text { types: } \\
\text { rockfall, } \\
\text { debris flow, } \\
\text { landslide, } \\
\text { avalanche, } \\
\text { flood, other }\end{array}$ & $\begin{array}{c}8 \text { types: } \\
\text { rockfall, } \\
\text { debris flow, } \\
\text { landslide, } \\
\text { avalanche, } \\
\text { flood, hail, } \\
\text { snowdrift, } \\
\text { falling tree }\end{array}$ & $\begin{array}{c}\text { 3 classes: } \\
\text { upstream, } \\
\text { downstream } \\
\text { and } \\
\text { unknown }\end{array}$ & $\begin{array}{c}4 \text { classes: } \\
\text { upstream, } \\
\text { downstream } \\
\text {, risk (no } \\
\text { event, only } \\
\text { preventive } \\
\text { closure) and } \\
\text { unknown }\end{array}$ & $\begin{array}{c}3 \text { classes: } \\
\text { near (few } \\
\text { meters to } 10 \\
\text { meters, far } \\
(>10 \mathrm{~m}) \text { or } \\
\text { prevention } \\
\text { (only } \\
\text { proventive } \\
\text { closure) }\end{array}$ & $\begin{array}{l}\text { Estimation } \\
\text { of the falled } \\
\text { volume on } \\
\text { the track of } \\
\text { the event }\end{array}$ & $\begin{array}{l}\text { Masse of the } \\
\text { event (only } \\
\text { for rockfall) }\end{array}$ & - & $\begin{array}{c}3 \text { classes: } \\
\text { small, } \\
\text { middle, big } \\
\text { (huge event) }\end{array}$ & - & - & - \\
\hline Source & $\begin{array}{l}\text { Online } \\
\text { article }\end{array}$ & $\begin{array}{l}\text { Online } \\
\text { article }\end{array}$ & $\begin{array}{l}\text { Online } \\
\text { article }\end{array}$ & $\begin{array}{l}\text { Online } \\
\text { article }\end{array}$ & $\begin{array}{l}\text { Online } \\
\text { article }\end{array}$ & $\begin{array}{l}\text { Online } \\
\text { article }\end{array}$ & $\begin{array}{l}\text { Online } \\
\text { article }\end{array}$ & $\begin{array}{l}\text { Online } \\
\text { article }\end{array}$ & $\begin{array}{l}\text { Online } \\
\text { article }\end{array}$ & $\begin{array}{l}\text { Online } \\
\text { article }\end{array}$ & $\begin{array}{l}\text { Online } \\
\text { article }\end{array}$ & $\begin{array}{l}\text { Online } \\
\text { article or } \\
\text { field visit }\end{array}$ \\
\hline
\end{tabular}




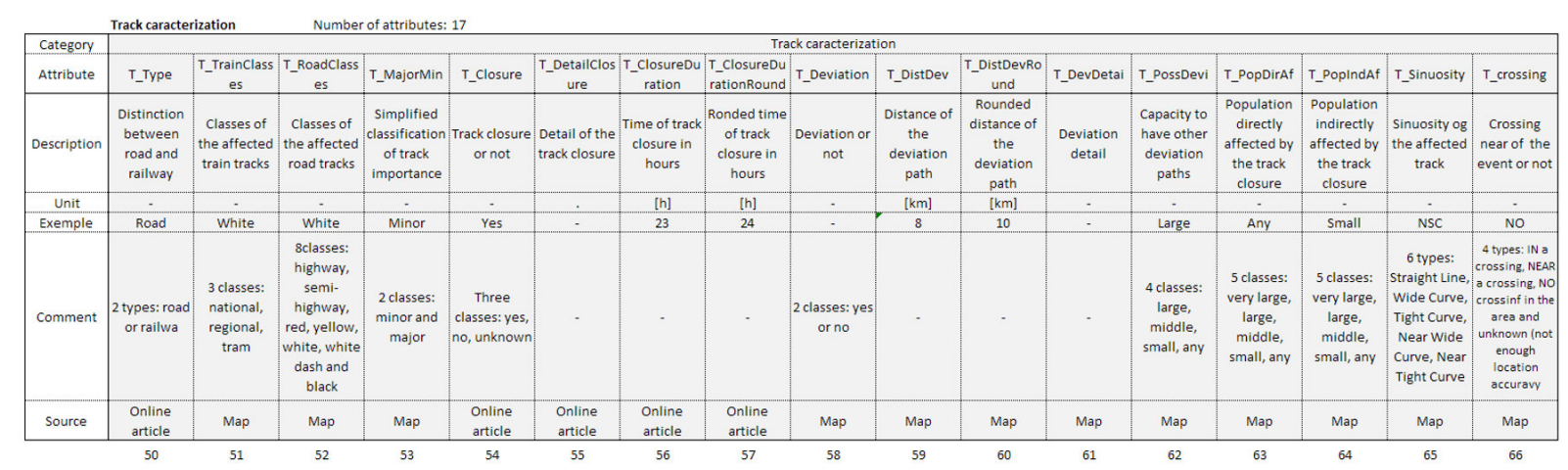

\begin{tabular}{|c|c|c|c|c|c|c|c|c|c|c|c|}
\hline Category & \multicolumn{11}{|c|}{ Damage } \\
\hline Attribute & D_Form & D_Injured & D_InjuredNb & D_Death & D_DeathNb & D_vehicule & D_ImpactTy & D_vehitype & D_Vehinb & D_TrackDeta & i| D_Infras_typ \\
\hline Description & $\begin{array}{c}\text { Form of track } \\
\text { damage }\end{array}$ & $\begin{array}{l}\text { Injured } \\
\text { people? }\end{array}$ & $\begin{array}{l}\text { Number of } \\
\text { injured } \\
\text { people }\end{array}$ & $\begin{array}{l}\text { Killed } \\
\text { people? }\end{array}$ & $\begin{array}{c}\text { Number of } \\
\text { killed } \\
\text { people }\end{array}$ & $\begin{array}{l}\text { Damage to } \\
\text { vehicle }\end{array}$ & \begin{tabular}{|c|} 
Type of \\
impact \\
between \\
vehicle and \\
event
\end{tabular} & $\begin{array}{l}\text { Type of } \\
\text { damaged } \\
\text { vehicle }\end{array}$ & $\begin{array}{c}\text { Number of } \\
\text { damaged } \\
\text { vehicle }\end{array}$ & $\begin{array}{l}\text { Detail of } \\
\text { track } \\
\text { damage }\end{array}$ & $\begin{array}{l}\text { Type of } \\
\text { instrastructu } \\
\text { re damage }\end{array}$ \\
\hline $\begin{array}{l}\text { Unit } \\
\text { Exemple }\end{array}$ & $\therefore$ & - & $\therefore$ & - & . & 5 &. & - & - & & - \\
\hline Comment & 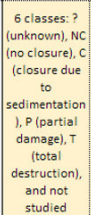 & $\begin{array}{l}2 \text { types: yes } \\
\text { or no }\end{array}$ & - & $\begin{array}{c}2 \text { types: yes } \\
\text { or no }\end{array}$ & - & $\begin{array}{c}2 \text { types: yes } \\
\text { or no }\end{array}$ & $\begin{array}{l}\text { Three types: } \\
\text { no impact, } \\
\text { direct impact } \\
\text { or indirect } \\
\text { impact }\end{array}$ & - & - & & - \\
\hline Source & $\begin{array}{l}\text { Online } \\
\text { article }\end{array}$ & $\begin{array}{l}\text { Online } \\
\text { article }\end{array}$ & $\begin{array}{l}\text { Online } \\
\text { article }\end{array}$ & $\begin{array}{l}\text { Online } \\
\text { article }\end{array}$ & $\begin{array}{l}\text { Online } \\
\text { article }\end{array}$ & $\begin{array}{l}\text { Online } \\
\text { article }\end{array}$ & $\begin{array}{l}\text { Online } \\
\text { article }\end{array}$ & $\begin{array}{l}\text { Online } \\
\text { article }\end{array}$ & $\begin{array}{l}\text { Online } \\
\text { article }\end{array}$ & & $\begin{array}{l}\text { Online } \\
\text { article }\end{array}$ \\
\hline
\end{tabular}
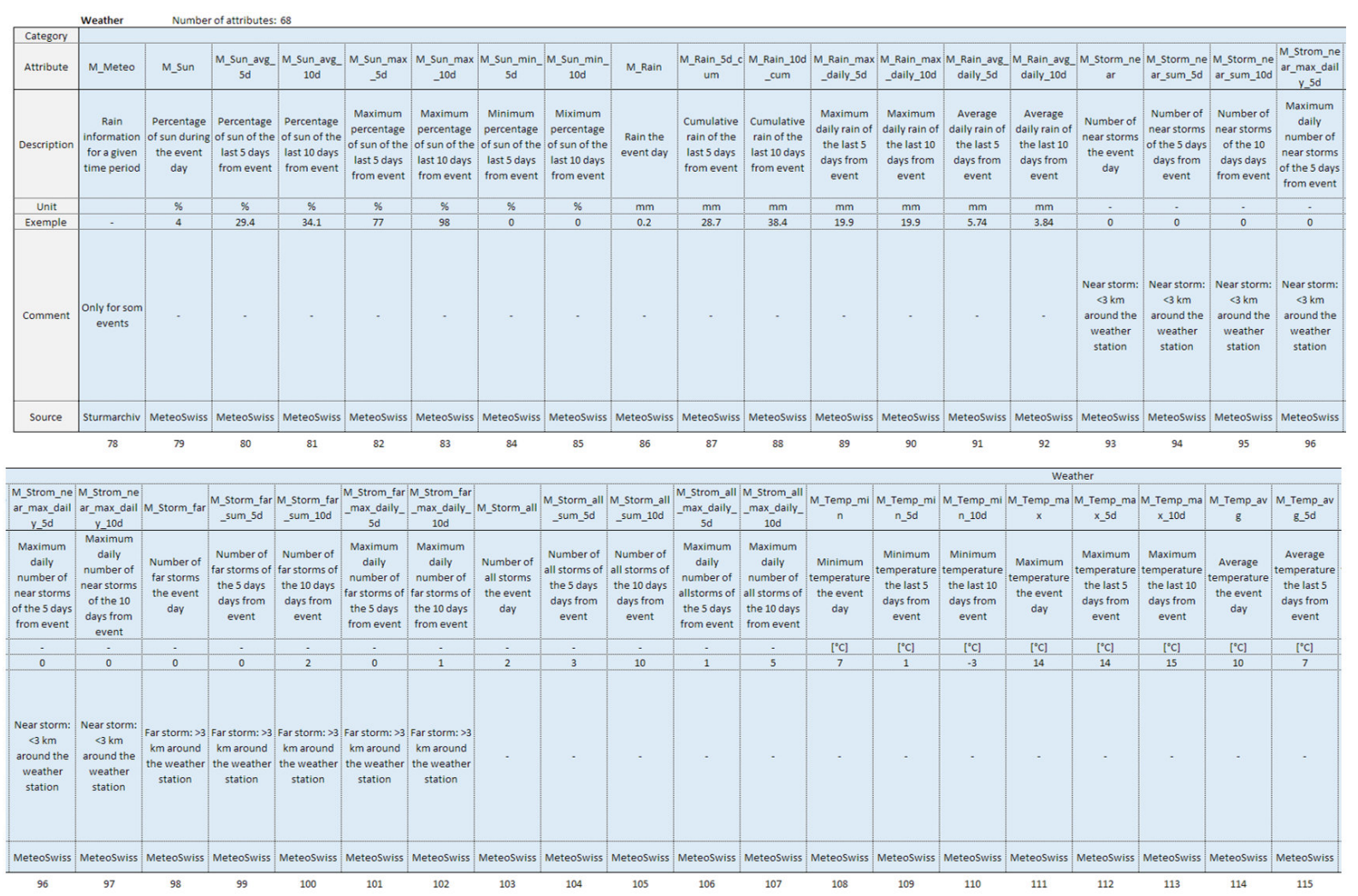


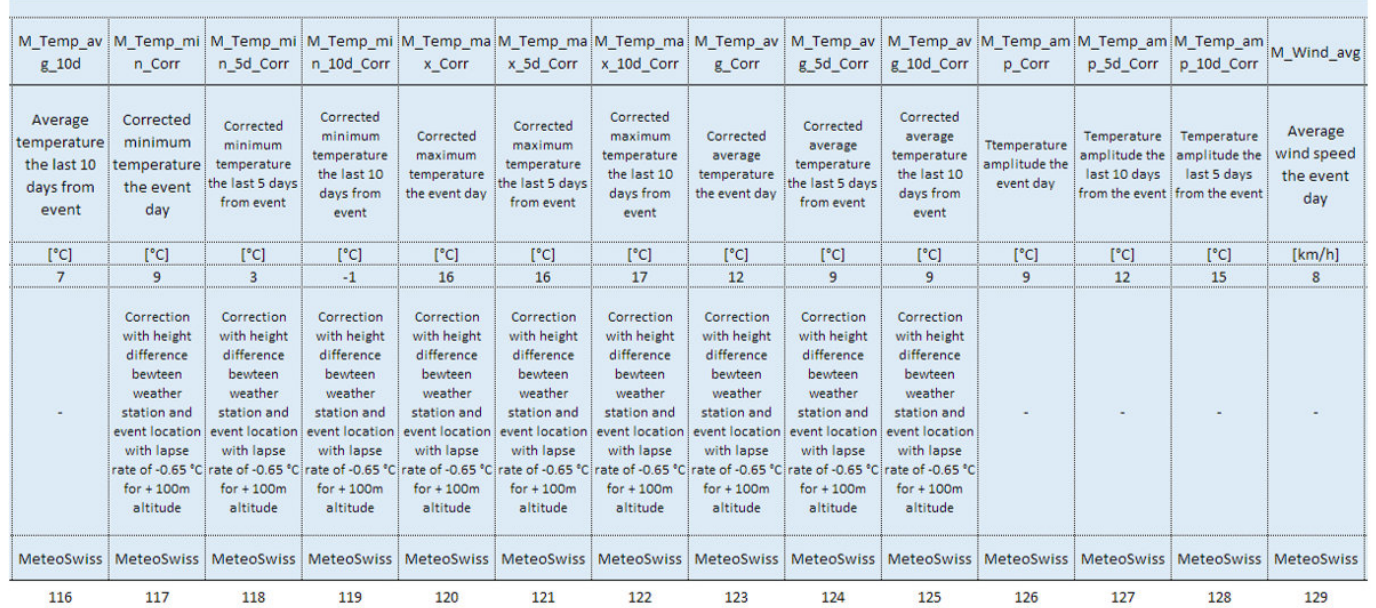

\begin{tabular}{|c|c|c|c|c|c|c|c|c|c|c|c|c|c|c|c|}
\hline $\begin{array}{c}\text { M_Wind_avg } \\
\text { _5d }\end{array}$ & $\begin{array}{c}\text { M_Win_avg_ } \\
10 d\end{array}$ & $\begin{array}{c}\text { M_Wind_ma } \\
x\end{array}$ & $\underset{\text { X_sd }}{\text { M_Wind_ma }}$ & $\begin{array}{c}\text { M_Wind_ma } \\
\text { x_10d }\end{array}$ & M_Wind_dir & $\underset{5 d}{M}$ & $\begin{array}{c}\text { M_Win_dir_ } \\
10 d\end{array}$ & M_Snow & $\begin{array}{c}\text { M_Fresh_sn } \\
\text { ow }\end{array}$ & $\begin{array}{c}\text { M_Fresh_sn } \\
\text { ow_5d }\end{array}$ & $\begin{array}{c}\text { M_Fresh_sn } \\
\text { ow_10d }\end{array}$ & $\begin{array}{l}\text { M_Accronym } \\
\text {-Stn_Weath }\end{array}$ & $\begin{array}{c}\text { M_Alt_Stn_ } \\
\text { Weath }\end{array}$ & $\begin{array}{c}\text { M_Diff_Alt_S } \\
\text { tn_Weath_E } \\
\text { vent }\end{array}$ & $\begin{array}{c}\text { M_Dist_Stn_ } \\
\text { Weath }\end{array}$ \\
\hline $\begin{array}{c}\text { Average } \\
\text { wind speed } \\
\text { the } 5 \text { last } \\
\text { days from } \\
\text { event }\end{array}$ & $\begin{array}{c}\text { Average } \\
\text { wind speed } \\
\text { the last } 10 \\
\text { days from } \\
\text { event }\end{array}$ & $\begin{array}{c}\text { Maximum } \\
\text { wind speed } \\
\text { the event } \\
\text { day }\end{array}$ & $\begin{array}{c}\text { Maximum } \\
\text { wind speed } \\
\text { the } 5 \text { last } \\
\text { days from } \\
\text { event }\end{array}$ & $\begin{array}{c}\text { Maximum } \\
\text { wind speed } \\
\text { the last } 10 \\
\text { days from } \\
\text { event }\end{array}$ & $\begin{array}{c}\text { Average } \\
\text { wind } \\
\text { direction the } \\
\text { event day }\end{array}$ & $\begin{array}{c}\text { Average } \\
\text { wind } \\
\text { direction the } \\
\text { last } 5 \text { days } \\
\text { from event }\end{array}$ & $\begin{array}{c}\text { Average } \\
\text { wind } \\
\text { direction the } \\
\text { last } 10 \text { days } \\
\text { from event }\end{array}$ & $\begin{array}{l}\text { Snow cover } \\
\text { height the } \\
\text { event day }\end{array}$ & $\begin{array}{c}\text { Fresh snow } \\
\text { cover height } \\
\text { the event } \\
\text { day }\end{array}$ & $\begin{array}{c}\text { Fresh snow } \\
\text { cover height } \\
\text { the } 5 \text { last } \\
\text { days from } \\
\text { event }\end{array}$ & $\begin{array}{c}\text { Fresh snow } \\
\text { cover height } \\
\text { the } 5 \text { last } \\
\text { days from } \\
\text { event }\end{array}$ & $\begin{array}{c}\text { Accronym of } \\
\text { the used } \\
\text { weather } \\
\text { station }\end{array}$ & $\begin{array}{c}\text { Altitude of } \\
\text { the used } \\
\text { weather } \\
\text { station }\end{array}$ & $\begin{array}{l}\text { Altitude } \\
\text { difference } \\
\text { between the } \\
\text { weather } \\
\text { station and } \\
\text { the even } \\
\text { location }\end{array}$ & $\begin{array}{l}\text { Distance } \\
\text { between the } \\
\text { weather } \\
\text { station and } \\
\text { the even } \\
\text { location }\end{array}$ \\
\hline$[\mathrm{km} / \mathrm{h}]$ & {$[\mathrm{km} / \mathrm{h}]$} & {$[\mathrm{km} / \mathrm{h}]$} & {$[\mathrm{km} / \mathrm{h}]$} & {$[\mathrm{km} / \mathrm{h}]$} & {$[\%]$} & {$[\%]$} & {$\left[{ }^{\circ}\right]$} & {$[\mathrm{cm}]$} & {$[\mathrm{cm}]$} & {$[\mathrm{cm}]$} & {$[\mathrm{cm}]$} & - & [m] a.s.I. & [m] & {$[\mathrm{km}]$} \\
\hline 9 & 10 & 32 & 38 & 46 & 47 & 48 & 63.9 & 0 & 0 & 0 & 0 & ZER & 1638 & -261 & 36 \\
\hline - & - & - & - & - & $\begin{aligned} 0^{\circ} & =\text { North }, \\
90^{\circ} & =\text { East }, \\
180^{\circ} & =\text { South }, \\
270^{\circ} & =\text { West }\end{aligned}$ & $\begin{aligned} 0^{\circ} & =\text { North }, \\
90^{\circ} & =\text { East }, \\
180^{\circ} & =\text { South } \\
270^{\circ} & =\text { West }\end{aligned}$ & $\begin{aligned} 0^{\circ} & =\text { North }, \\
90^{\circ} & =\text { East }, \\
180^{\circ} & =\text { South }, \\
270^{\circ} & =\text { West }\end{aligned}$ & - & - & - & - & - & - & - & - \\
\hline MeteoSwiss & MeteoSwiss & MeteoSwiss & MeteoSwiss & Meteoswiss & MeteoSwiss & MeteoSwiss & MeteoSwiss & Meteoswiss & MeteoSwiss & MeteoSwiss & Meteoswiss & MeteoSwiss & Meteoswiss & Meteoswiss & Meteoswiss \\
\hline
\end{tabular}

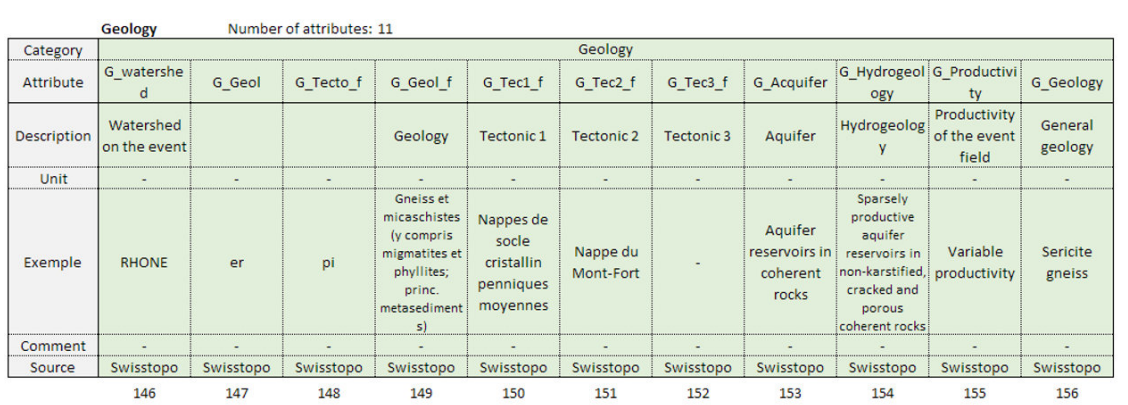

\begin{tabular}{|c|c|c|c|c|c|c|c|c|c|c|c|c|c|c|c|c|}
\hline \multirow{2}{*}{\begin{tabular}{|l|} 
Category \\
Attribute \\
\end{tabular}} & \multicolumn{16}{|c|}{ Source } \\
\hline & Source1 & Source2 & Source 3 & Source4 & Sources & Source6 & Source7 & Sources & Sources & Source10 & Sourcel1 & Source12 & Source13 & Source14 & Source15 & Source16 \\
\hline Description & $\begin{array}{l}\text { Source } 1 \text { for } \\
\text { the event }\end{array}$ & $\begin{array}{l}\text { Source } 2 \text { for } \\
\text { the event }\end{array}$ & $\begin{array}{c}\text { Source } 3 \text { for } \\
\text { the event }\end{array}$ & $\begin{array}{c}\text { Source } 4 \text { for } \\
\text { the event }\end{array}$ & $\begin{array}{l}\text { Source } 5 \text { for } \\
\text { the event }\end{array}$ & $\begin{array}{c}\text { Source } 6 \text { for } \\
\text { the event }\end{array}$ & $\begin{array}{l}\text { Source } 7 \text { for } \\
\text { the event }\end{array}$ & $\begin{array}{l}\text { Source } 8 \text { for } \\
\text { the event }\end{array}$ & $\begin{array}{l}\text { Source } 9 \text { for } \\
\text { the event }\end{array}$ & $\begin{array}{l}\text { Source } 10 \mathrm{for} \\
\text { the event }\end{array}$ & $\begin{array}{l}\text { Source } 11 \text { for } \\
\text { the event }\end{array}$ & $\begin{array}{l}\text { Source } 12 \text { for } \\
\text { the event }\end{array}$ & $\begin{array}{l}\text { Source } 13 \text { for } \\
\text { the event }\end{array}$ & $\begin{array}{l}\text { Source } 14 \text { for } \\
\text { the event }\end{array}$ & $\begin{array}{l}\text { Source } 15 \text { for } \\
\text { the event }\end{array}$ & $\begin{array}{l}\text { Source } 16 \text { for } \\
\text { the event }\end{array}$ \\
\hline Unit & - & & $\therefore$ & - & - & - & - &. & - & - & - & - & - & - & - & - \\
\hline Exemple & 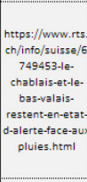 & 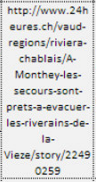 & 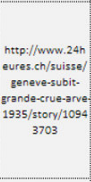 & 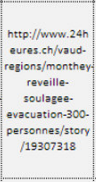 & 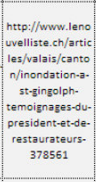 & 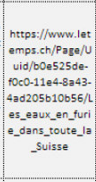 & 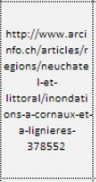 & 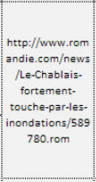 &  & 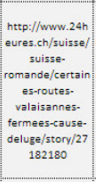 & 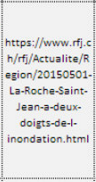 & 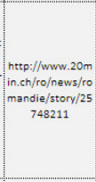 & - & - & - & - \\
\hline Comment & & & & & & & & & & & & & & & & \\
\hline Source & $\begin{array}{l}\text { Google } \\
\text { Alerts }\end{array}$ & $\begin{array}{l}\text { Google } \\
\text { Alerts }\end{array}$ & $\begin{array}{l}\text { Google } \\
\text { Alerts }\end{array}$ & $\begin{array}{l}\text { Google } \\
\text { Alerts }\end{array}$ & $\begin{array}{l}\text { Google } \\
\text { Alerts }\end{array}$ & $\begin{array}{l}\text { Google } \\
\text { Alerts }\end{array}$ & $\begin{array}{l}\text { Google } \\
\text { Alerts }\end{array}$ & $\begin{array}{c}\text { Google } \\
\text { Alerts }\end{array}$ & $\begin{array}{l}\text { Google } \\
\text { Alerts }\end{array}$ & $\begin{array}{l}\text { Google } \\
\text { Alerts }\end{array}$ & $\begin{array}{l}\text { Google } \\
\text { Alerts }\end{array}$ & $\begin{array}{l}\text { Google } \\
\text { Alerts }\end{array}$ & $\begin{array}{l}\text { Google } \\
\text { Alerts }\end{array}$ & $\begin{array}{l}\text { Google } \\
\text { Alerts }\end{array}$ & $\begin{array}{l}\text { Google } \\
\text { Alerts }\end{array}$ & $\begin{array}{l}\text { Google } \\
\text { Alerts }\end{array}$ \\
\hline & 157 & 158 & 159 & 160 & 161 & 162 & 163 & 164 & 165 & 166 & 167 & 168 & 169 & 170 & 171 & 172 \\
\hline
\end{tabular}



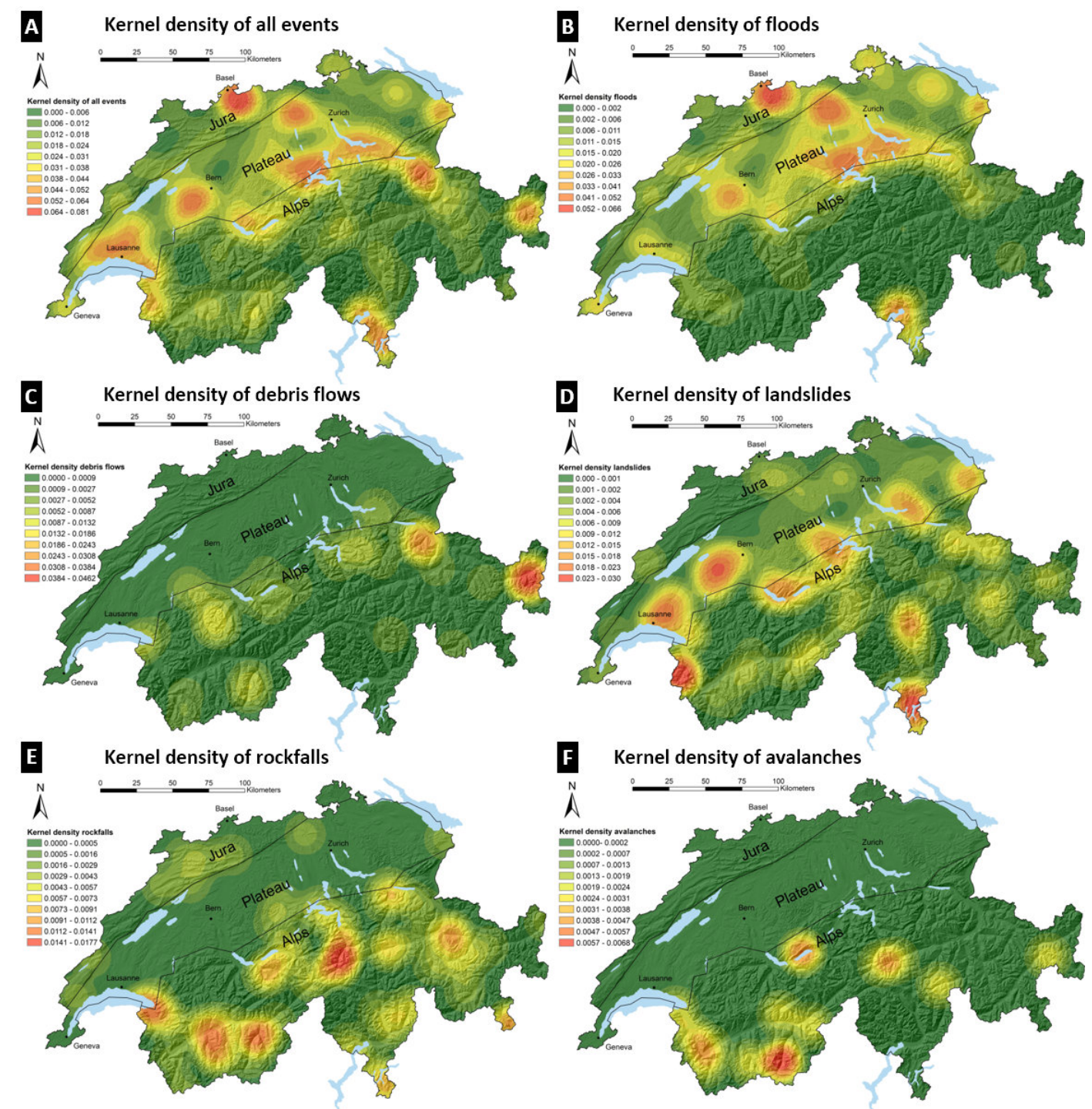

F Kernel density of avalanches
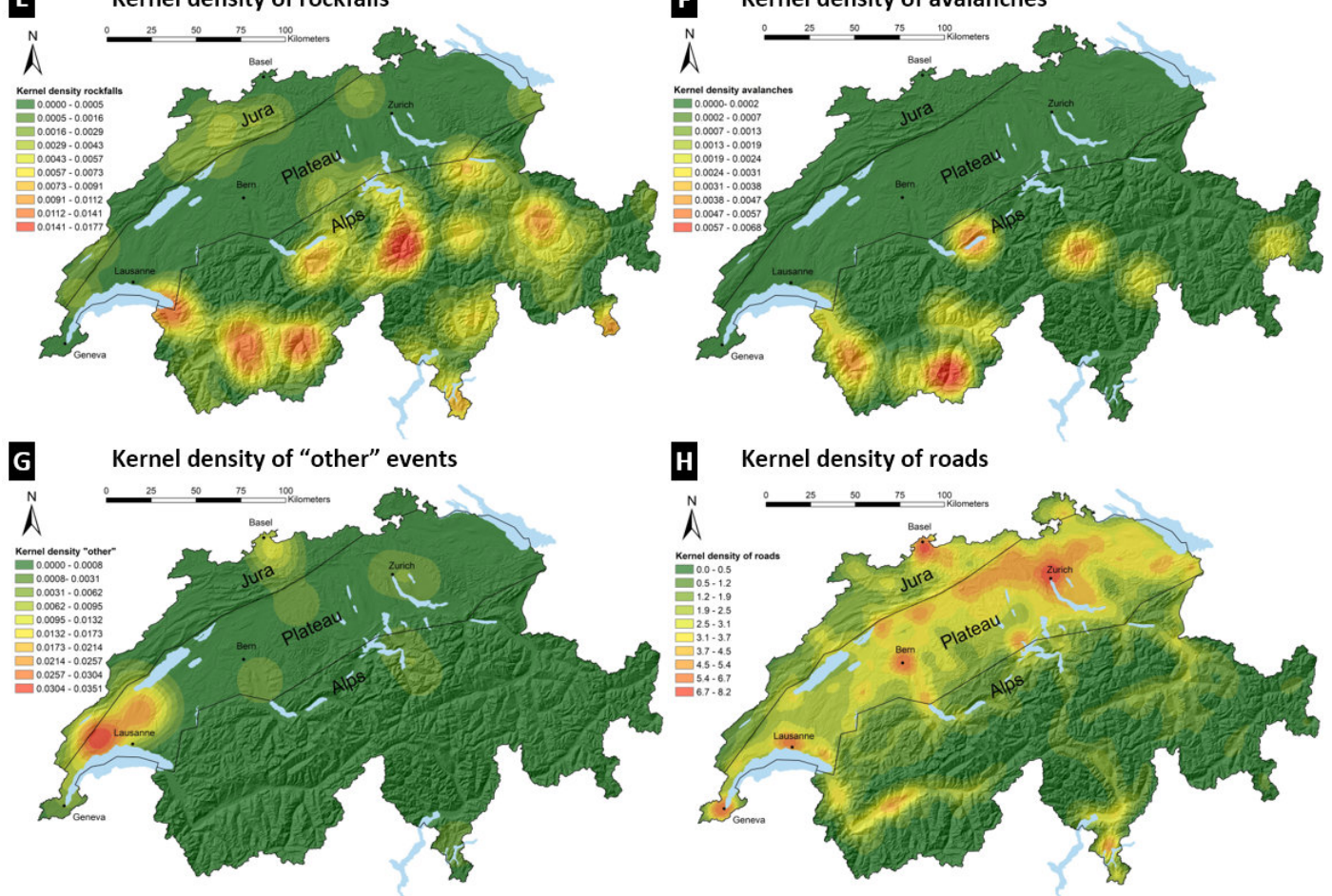

Figure S2: Kernel density maps. Search radius for events: $20 \mathrm{~km}$. Search radius for road network: $10 \mathrm{~km}$. The results were classified using 10 classes with the Jenks natural breaks method. A: All events; B: Floods; $C$ : Debris flows; D: Landslides; E: Rockfalls; F: Avalanches; G: "Other"; H: Roads. Hillshade and map ground sources: Swisstopo. 


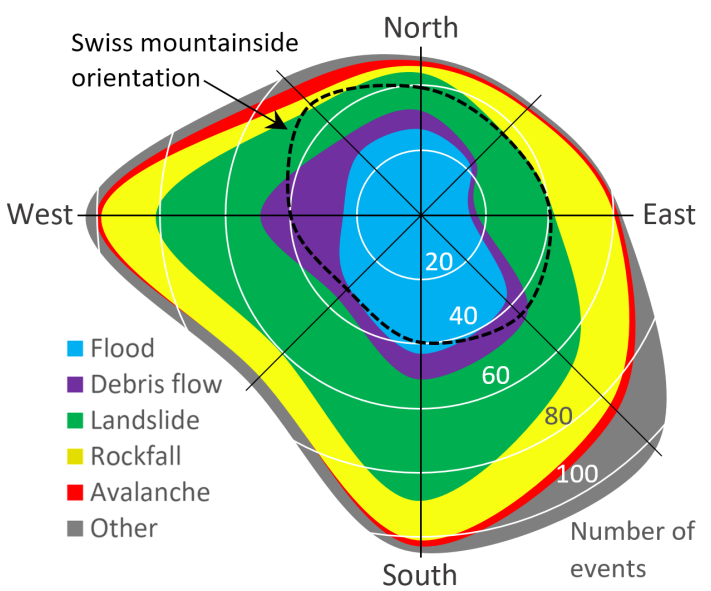

Figure S3: Slope orientation distribution of natural hazard events on the Swiss transportation network from 2012 to 2016. The relative distribution of Swiss mountainside orientation is shown by the black dashed line.

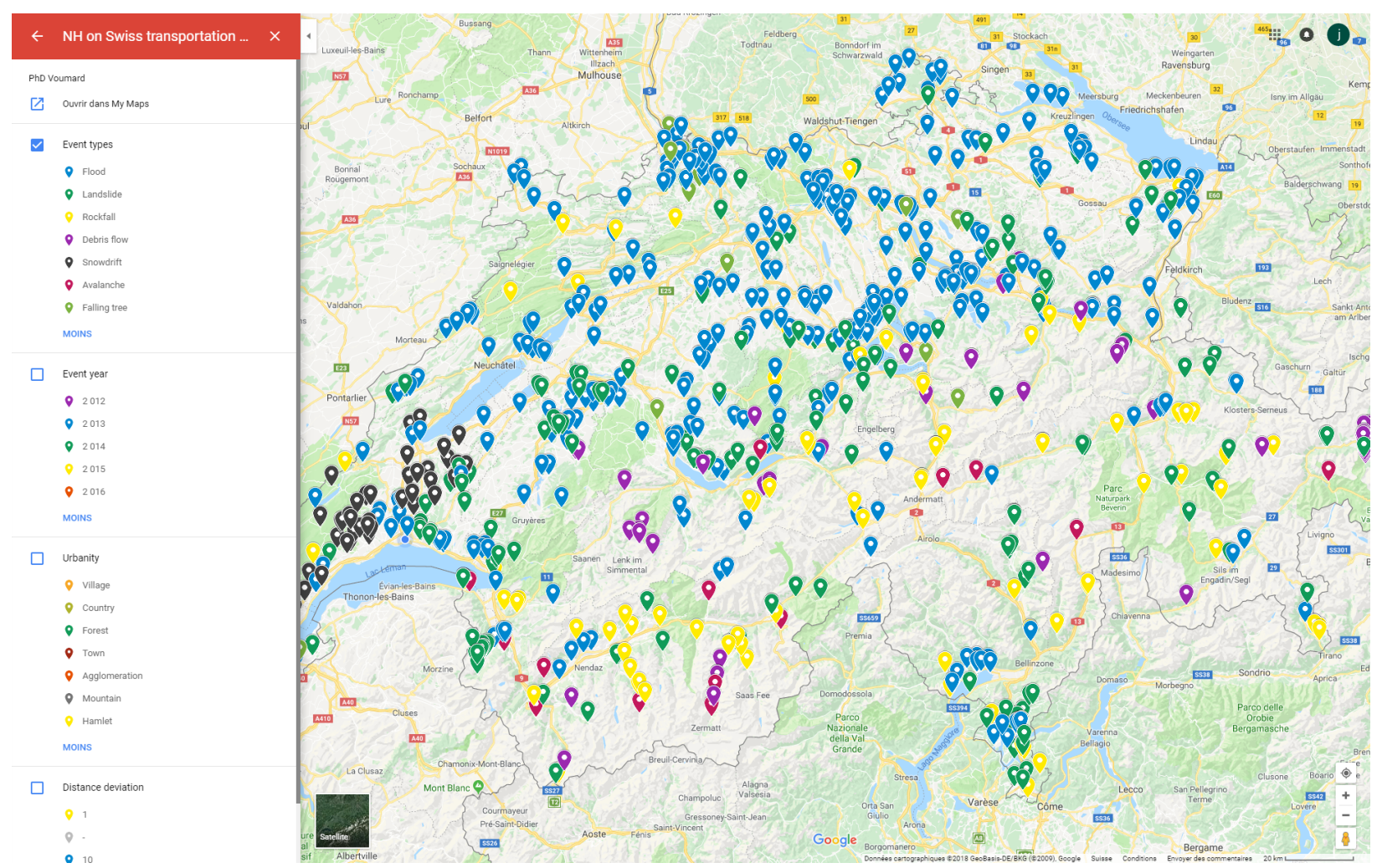

Figure S4: Database on Google Maps. Available at (last accessed: 25 January 2018):

94 https://www.google.ch/maps/@46.7199391,7.1246016,8z/data=!4m2!6m1!1s1qtu6LEYum-

$957 \mathrm{ghpPg} 9 \mathrm{WWzWwgPHYA}$ ?hl=fr, last access: 25 January 2018. 




Figure S5: Database on ArcGIS online. Available at (last accessed: 25 January 2018):

99 http://unil.maps.arcgis.com/apps/MapTools/index.html?webmap=34ee3eb719a647889abd34175969d781, last

100 access: 25 January 2018. 